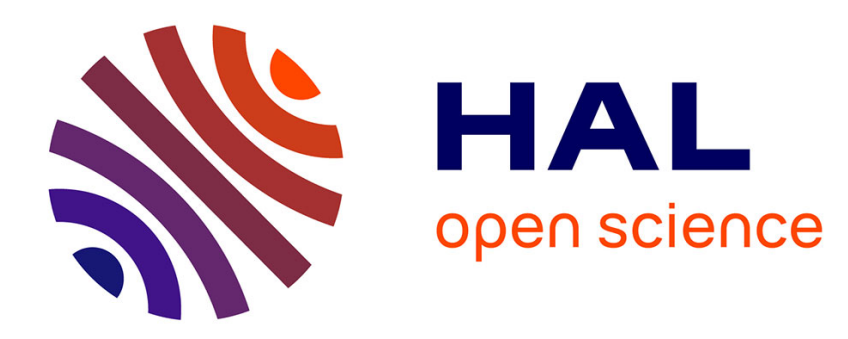

\title{
Singular solutions of the BBM equation: analytical and numerical study
}

Sergey Gavrilyuk, Keh-Ming Shyue

\section{To cite this version:}

Sergey Gavrilyuk, Keh-Ming Shyue. Singular solutions of the BBM equation : analytical and numerical study. 2021. hal-03220465

\section{HAL Id: hal-03220465 \\ https://hal.science/hal-03220465}

Preprint submitted on 7 May 2021

HAL is a multi-disciplinary open access archive for the deposit and dissemination of scientific research documents, whether they are published or not. The documents may come from teaching and research institutions in France or abroad, or from public or private research centers.
L'archive ouverte pluridisciplinaire HAL, est destinée au dépôt et à la diffusion de documents scientifiques de niveau recherche, publiés ou non, émanant des établissements d'enseignement et de recherche français ou étrangers, des laboratoires publics ou privés. 


\title{
Singular solutions of the BBM equation : analytical and numerical study
}

\author{
Sergey Gavrilyuk, Keh-Ming Shyue ${ }^{\dagger}$
}

May 7, 2021

\begin{abstract}
We show that the Benjamin-Bona-Mahony (BBM) equation admits stable travelling wave solutions representing a sharp transition front linking a constant state with a periodic wave train. The constant state is determined by the parameters of the periodic wave train : the wave length, amplitude and phase velocity, and satisfies both the Rankine-Hugoniot conditions for the corresponding Whitham modulation system and generalized Rankine-Hugoniot conditions for the exact BBM equation. Such stable shock-like travelling structures exist if the phase velocity of the periodic wave train is not less than the solution average value. To validate the accuracy of the numerical method, we derive the (singular) solitary limit of the Whitham system for the BBM equation and compare the corresponding numerical and analytical solutions. We find good agreement between analytical results and numerical solutions.
\end{abstract}

Keyword : nonlinear dispersive equations, shock-like transition fronts, Whitham's modulation equations, solitary limit

Mathematics Subject Classification numbers: 35L40, 35Q35, 35Q74.

\section{Introduction}

The Benjamin-Bona-Mahony (BBM) equation was proposed as a unidirectional model of weakly non-linear waves in shallow water [5]:

$$
v_{t}+v_{x}+v v_{x}-v_{t x x}=0,
$$

involving one dependent variable $v(t, x)$ and two independent variables $t$ (time) and $x$ (space coordinate). The last term $v_{t x x}$ is responsible for the nonlocal

\footnotetext{
*Aix Marseille Univ, CNRS, IUSTI, UMR 7343, Marseille, France, sergey.gavrilyuk@univamu.fr

${ }^{\dagger}$ Institute of Applied Mathematical Sciences, National Taiwan University, Taipei 106, Taiwan, shyue@ntu.edu.tw
} 
nature of the BBM equation. After the change of variables $v=u-1$ one gets the equation :

$$
u_{t}+u u_{x}-u_{t x x}=0 .
$$

P. Olver [30] justified that (1) admits only three independent conservation laws:

$$
\begin{gathered}
\left(u-u_{x x}\right)_{t}+\left(\frac{u^{2}}{2}\right)_{x}=0, \\
\left(\frac{u^{2}}{2}+\frac{u_{x}^{2}}{2}\right)_{t}+\left(\frac{u^{3}}{3}-u u_{t x}\right)_{x}=0 \\
\left(\frac{u^{3}}{3}\right)_{t}-\left(u_{t}^{2}-u_{x t}^{2}+u^{2} u_{x t}-\frac{u^{4}}{4}\right)_{x}=0,
\end{gathered}
$$

and proposed a Hamiltonian formulation of the BBM equation [31]. In particular, (1) can be written as the Euler-Lagrange equation for the Lagrangian

$$
\mathcal{L}=-\frac{\varphi_{t} \varphi_{x}}{2}+\frac{\varphi_{t} \varphi_{x x x}}{2}-\frac{\varphi_{x}^{3}}{6}, \quad u=\varphi_{x}
$$

A number of important qualitative results have been obtained for the BBM equation : in [39] the modulation equations were derived; the well (ill)-posedness of the Cauchy problem for the BBM equation was studied in [2]; the modulational instability of short periodic waves has been proven in [29].

The Riemann problem for the BBM equation is the Cauchy problem

$$
u(0, x)= \begin{cases}u^{-}, & x<0, \\ u^{+}, & x>0 .\end{cases}
$$

with constant values of $u^{ \pm}$. Such a problem is often called Gurevich-Pitaevskii problem, who were the first to give its asymptotic solution for the Kortewegde Vries (KdV) equation [20]. This approach has been further developed and applied to both integrable and non-integrable dispersive equations $[21,17,10$, $11,12,23,3,4]$. The Riemann problem for (1) was recently investigated in [8]. The authors analytically and numerically studied the influence of the initial step data and of a smoothing parameter (the stepwise initial data was replaced by the hyperbolic tangent having this parameter as a characteristic width of the transition zone) on the solution structure. The fact that the solution can depend on the smoothing parameter has been also discussed in [35] for the Serre-GreenNaghdi (SGN) equations which is a nonlinear bi-directional model of shallow water flows [36, 18, 19, 34].

The BBM equation admits exact weak stationary solutions which are at the same time weak solutions to the Hopf equation $u_{t}+\left(u^{2} / 2\right)_{x}=0$ [12]. In particular, for the antisymmetric initial data $u^{+}=-u^{-}<0$ the solution is a shock satisfying Lax entropy condition, while $u^{+}=-u^{-}>0$ corresponds to an unstable shock which transforms to a rarefaction wave (which is also a solution to both the BBM and Hopf equations). Numerically, the Lax shock is accompanied by narrow zones of very short waves. The shock solution is not 
structurally stable under non-symmetric perturbations. For $u^{+}=-u^{-}>0$, a transient discontinuous structure appears decaying algebraically in time and finally degenerating into the rarefaction wave of the Hopf equation [13, 8].

A natural question appears whether non-transient discontinuous structures can appear as stable solutions of the BBM equation. Such shock-like structures were recently discovered for the SGN equations and linear Boussinesq equations [14]. They appeared as solutions of the generalized Riemann problem where constant initial states were replaced by periodic solutions of the SGN equations. In particular, the authors of [14] found such shock-like transition fronts linking a constant state with a periodic wave train. The velocity of such a shock coincides with the velocity of the periodic wave train. Across the shock considered as a dispersionless limit, generalized Rankine-Hugoniot conditions were satisfied. These conditions are the classical conservation laws for mass and momentum augmented by an additional condition which expresses the continuity of one-sided first order derivatives of unknowns. Physically, this extra condition is nothing but the absence of oscillations at the shock front (the onesided gradients of unknowns are vanishing). A multi-dimensional version of the generalized Rankine-Hugoniot conditions was also derived for a class of EulerLagrange equations describing, in particular, the second gradient fluid and fluids containing gas bubbles [15].

The question about the existence of shock-like transition fronts for the BBM equation is thus reasonable because the BBM and SGN equations share a common "hyperbolic" feature: the phase and group velocity obtained for the corresponding linearized equations are finite for any wave number.

One has to mention the result of [38] where the fifth order KdV equation was studied. The heteroclinic connection of the periodic orbits for the exact dispersive equation corresponded to the shocks for the Whitham system. Such a scenario cannot obviously appear for the BBM equation because the periodic solutions of the BBM equation are described by a low order Hamiltonian differential equation which does not admit periodic-to periodic or periodic-to constant connections.

The aim of this paper is to give precise conditions for the existence of stable shock-like structures for the BBM equation. To validate the accuracy of numerical results, we need to test the numerical method (see a short description in Appendix B) on closed form analytical solutions (e.g., travelling waves) or asymptotic solutions (e.g., the solutions of modulation equations for the BBM equation). The test based on travelling wave solution is a little bit trivial. It is interesting thus to find closed form analytical non-stationary solutions of the modulation equations (three equations model), but they do not exist in the literature. Indeed, the BBM equation is not integrable, so no hope to rewrite the modulation equations in the form of Riemann invariants, as it was done for the $\mathrm{KdV}$ equation [41] and NLS equation [32]. One of the possibilities is to find the solitary limit of the corresponding modulation equations. For this we need to find a long wave limit of the wave action conservation law [17]. For generic Hamiltonian systems such an approach was recently developed in [6] with interesting applications to the second gradient fluids. We will derive such a solitary 
limit for the BBM equation and will obtain corresponding analytical solutions.

\section{Periodic solutions of the BBM equation}

The travelling wave solutions of the BBM equation $u=u(\xi), \xi=x-D t$ satisfy the equation:

$$
-D\left(u-u^{\prime \prime}\right)+\frac{u^{2}}{2}=c_{1}, \quad c_{1}=\text { const. }
$$

Here "prime" means the derivative with respect to $\xi$. It implies the first integral:

$$
D \frac{u^{\prime 2}}{2}=-\frac{u^{3}}{6}+D \frac{u^{2}}{2}+c_{1} u+c_{2}=\frac{1}{6}\left(u-u_{1}\right)\left(u-u_{2}\right)\left(u_{3}-u\right), \quad c_{2}=\mathrm{const},
$$

where new constants $u_{1} \leq u_{2} \leq u_{3}$ are introduced. They related with $D, c_{1}$, and $c_{2}$ :

$$
D=\frac{1}{3}\left(u_{1}+u_{2}+u_{3}\right), \quad c_{1}=-\frac{1}{6}\left(u_{1} u_{2}+u_{1} u_{3}+u_{2} u_{3}\right), \quad c_{2}=\frac{1}{6} u_{1} u_{2} u_{3} .
$$

Another form of the equation is :

$$
\left(u_{1}+u_{2}+u_{3}\right) u^{\prime 2}=P(u), \quad P(u)=\left(u-u_{1}\right)\left(u-u_{2}\right)\left(u_{3}-u\right) .
$$

In the following, we will consider only positive solutions $\left(0<u_{1}<u_{2}<u<u_{3}\right)$ (the negative solutions can be found by the symmetry $u \rightarrow-u$ and $D \rightarrow-D$ ). The periodic solution $u(x)$ is given by :

$$
u(x)=u_{2}+a \mathrm{cn}^{2}(\eta, m),
$$

where

$$
m=\frac{u_{3}-u_{2}}{u_{3}-u_{1}}, \quad a=u_{3}-u_{2}, \quad \eta=\frac{\xi+\xi_{0}}{2 \sqrt{3 D}} \sqrt{\frac{a}{m}}, \quad \xi_{0}=\text { const. }
$$

Here $\operatorname{cn}(\eta, m)=\cos (\varphi(\eta, m))$, where $\varphi$ is defined implicitly from

$$
\eta=\int_{0}^{\varphi(\eta, m)} \frac{d \theta}{\sqrt{1-m \sin ^{2} \theta}} .
$$

The wave length is given as

$$
L=4 \sqrt{3} \sqrt{\frac{D m}{a}} K(m) .
$$

In particular, the solitary wave solution obtained in the limit $L \rightarrow \infty$ and for the values $u_{1}=u_{2}>0, a=u_{3}-u_{2}$ is in the form

$$
u(\xi)=u_{2}+\frac{a}{\cosh ^{2}(\eta)}, \quad \eta=\frac{\xi+\xi_{0}}{2 \sqrt{1+\frac{3 u_{2}}{a}}}, \quad D=u_{2}+\frac{a}{3}, \quad \xi_{0}=\text { const. }
$$


We will define the average value of any function $f(u)$ as

$$
\overline{f(u)}=\int_{u_{2}}^{u_{3}} \frac{f(u) d u}{\sqrt{P(u)}} / \int_{u_{2}}^{u_{3}} \frac{d u}{\sqrt{P(u)}} .
$$

In particular, the average value of $u$ (denoted below by $\bar{u}$ ) is given by :

$$
\bar{u}=\frac{\int_{u_{2}}^{u_{3}} \frac{u d u}{\sqrt{P(u)}}}{\int_{u_{2}}^{u_{3}} \frac{d u}{\sqrt{P(u)}}}=u_{1}+\left(u_{3}-u_{1}\right) \frac{E(m)}{K(m)}=u_{2}+\frac{a}{m}\left(\frac{E(m)}{K(m)}+m-1\right) .
$$

Here the complete elliptic integrals of the first and second type are defined as [1] :

$$
K(m)=\int_{0}^{\pi / 2} \frac{d \theta}{\sqrt{1-m \sin ^{2} \theta}}, \quad E(m)=\int_{0}^{\pi / 2} \sqrt{1-m \sin ^{2} \theta} d \theta .
$$

The inverse formulas expressing $u_{1}, u_{2}$ and $u_{3}$ as functions of $\bar{u}, a$ and $m$ are given by

$u_{1}=\bar{u}-\frac{a}{m} \frac{E(m)}{K(m)}, u_{2}=\bar{u}-\frac{a}{m}\left(\frac{E(m)}{K(m)}+m-1\right), u_{3}=\bar{u}-\frac{a}{m}\left(\frac{E(m)}{K(m)}-1\right)$.

One can check that the change of variables is invertible, i.e., its Jacobian matrix has its inverse because

$$
\operatorname{det}\left(\frac{\partial\left(u_{1}, u_{2}, u_{3}\right)}{\partial(\bar{u}, a, m)}\right)=\frac{a}{m^{2}} \neq 0 .
$$

The determinant becomes singular in the solitary limit $m \rightarrow 1$. The velocity $D$ is given by the formula

$$
D=\frac{1}{3}\left(u_{1}+u_{2}+u_{3}\right)=\bar{u}+\frac{a}{m}\left(\frac{2-m}{3}-\frac{E(m)}{K(m)}\right) .
$$

We will show further the importance of a special case $D=\bar{u}$ : the phase velocity coincides with the characteristic of the Hopf equation for the homogeneous state $\bar{u}$. The corresponding value of $m$ is the solution of :

$$
\frac{2-m}{3}=\frac{E(m)}{K(m)} \text {. }
$$

This value is unique : $m=m_{c} \approx 0.961149$.

\section{Whitham modulation equations for the BBM system}

Two equivalent methods can be used to obtain the modulation equations : the averaging of the conservation laws $[40,7]$ and Whitham's method of averaged 
Lagrangian [41]. Both methods are complementary in the analysis of the modulation equations. The first one assures the initial conservative structure of the governing equations, while the second one can give an idea about the choice of "appropriate" variables for the theoretical study of the modulation equations $[41,23]$.

The method of conservation laws for the BBM equation was used, in particular, in [39]. The essence of the method is as follows. We are looking for the solution $u(\xi, X, T, \varepsilon)$ which is periodic with respect to $\xi$ and varies slowly with respect to time and space, with $\xi=\frac{X-D T}{\varepsilon}=x-D t, \quad X=\varepsilon x, T=\varepsilon t, \varepsilon$ being a small parameter. The solution period $L$ is thus also a slowly varying function. Commuting the averaging with respect to $\xi$, and time and space derivatives, we obtain from the first two conservation laws (2)-(3) the equations :

$$
\begin{gathered}
(\bar{u})_{t}+\left(\frac{\overline{u^{2}}}{2}\right)_{x}=0 \\
\left(\frac{\overline{u^{2}}}{2}+\frac{\overline{u^{\prime 2}}}{2}\right)_{t}+\left(\frac{\overline{u^{3}}}{3}-D \overline{u^{\prime 2}}\right)_{x}=0 .
\end{gathered}
$$

We used here the relation $\overline{u^{\prime \prime}}=0, \overline{\left(u u^{\prime}\right)^{\prime}}=0$. The averaging of the third equation is equivalent to the phase conservation law [39] :

$$
k_{t}+(D k)_{x}=0, \quad k=\frac{1}{L} .
$$

For simplicity, we defined here the wave number $k$ as $1 / L$ and not as $2 \pi / L$. Also, instead of the slow variables $T, X$ we returned back to the variables $t, x$.

Using (10), one can write the modulation equations in an equivalent form :

$$
\begin{gathered}
(\bar{u})_{t}+\left(\frac{\overline{u^{2}}}{2}\right)_{x}=0 \\
\left(\frac{\overline{u^{2}}}{2}+\frac{\overline{P(u)}}{6 D}\right)_{t}+\left(\frac{\overline{u^{3}}}{3}-\frac{\overline{P(u)}}{3}\right)_{x}=0 \\
(1 / L)_{t}+(D / L)_{x}=0 .
\end{gathered}
$$

We choose the variables $\bar{u}, a$ and $m$ as unknowns. One can find :

$$
\begin{aligned}
\bar{u} & =u_{2}+a A_{1}, \\
\overline{u^{2}} & =u_{2}^{2}+2 u_{2} a A_{1}+a^{2} A_{2}=\bar{u}^{2}+a^{2}\left(A_{2}-A_{1}^{2}\right), \\
\overline{u^{3}} & =u_{2}^{3}+3 u_{2}^{2} a A_{1}+3 u_{2} a^{2} A_{2}+a^{3} A_{3} \\
& =\bar{u}^{3}+3 \bar{u} a^{2}\left(A_{2}-A_{1}^{2}\right)+a^{3}\left(A_{3}-3 A_{1} A_{2}+2 A_{1}^{3}\right), \\
\overline{P(u)} & =\frac{a^{3}}{m} P_{2}(m),
\end{aligned}
$$


with

$$
\begin{aligned}
& A_{k}(m)=\int_{0}^{\pi / 2} \frac{\cos ^{2 k} \theta d \theta}{\sqrt{1-m \sin ^{2} \theta}} / \int_{0}^{\pi / 2} \frac{d \theta}{\sqrt{1-m \sin ^{2} \theta}}, \\
& P_{2}(m)=\int_{0}^{\pi / 2} \sin ^{2} \theta \cos ^{2} \theta \sqrt{1-m \sin ^{2} \theta} d \theta / \int_{0}^{\pi / 2} \frac{d \theta}{\sqrt{1-m \sin ^{2} \theta}} .
\end{aligned}
$$

The integrals $A_{k}(m)$ and $P_{2}(m)$ can also be expressed in terms of $E(m)$ and $K(m)$ (see Appendix A). Still, even if the equations can now be explicitly written in terms of $a, \bar{u}, m$, it is difficult to extract from (23)-(25) "reasonably simple" closed form solutions to compare with numerical solutions of the exact BBM equation. The idea is to find the solitary limit of $(23),(24),(25)[17,6]$. This limit is singular, and we cannot obtain this limit directly from the three above written conservation laws. Indeed, (23)-(24) give in the limit the Hopf equation, while the equation (25) becomes a trivial identity. For this, we need to find a "non-obvious" additional conservation law (the action conservation law) of the modulation equations. It can be easily found by the Whitham method of averaged Lagrangian.

The Whitham method of the averaged Lagrangian consists in looking for a solution of the Euler-Lagrange equations for (5) of the form [41] :

$$
\varphi=\beta x-\gamma t+\psi(\theta), \quad \theta=k x-\omega t,
$$

with $\beta, \gamma, k, \omega$ depending on $T$ and $X$. The following relations are the compatibility conditions :

$$
\beta_{t}+\gamma_{x}=0, \quad k_{t}+\omega_{x}=0 .
$$

The function $\psi(\theta, T, X, \varepsilon)$ is supposed to be 1 -periodic with respect to the variable $\theta$. The variables $\theta$ and $\xi$ are related: $\theta=k \xi$.

The unknown functions should be determined as solutions of the EulerLagrange equations for the averaged Lagrangian

$$
\overline{\mathcal{L}}=\int_{0}^{1} \mathcal{L} d \theta
$$

where $\mathcal{L}$ is given by (5). The derivation is quite standard and follows directly the derivation of the modulation equations for the KdV equation (see [41], Section 16.14). We present here a rapid derivation. In zero order one has :

$$
\begin{aligned}
u & =\varphi_{x} \approx \beta+k \psi_{\theta}, \\
\varphi_{t} & \approx-\gamma-\omega \psi_{\theta}=-\gamma-D(u-\beta), \quad D=\frac{\omega}{k}, \\
\varphi_{x x x} & =u_{x x} \approx k^{2} u_{\theta \theta} .
\end{aligned}
$$

Then the zero order Lagrangian (5) (defined up to the full derivative with respect to $\theta)$ is :

$$
\mathcal{L} \approx \frac{u(\gamma-D \beta)}{2}+\frac{D u^{2}}{2}-\frac{u^{3}}{6}+\frac{D}{2} k^{2} u_{\theta}^{2}
$$


The dependence of $u$ on the rapid variable is determined from (7):

$$
D k^{2} u_{\theta \theta}-D u+\frac{u^{2}}{2}=c_{1} .
$$

It can be integrated once :

$$
D k^{2} \frac{u_{\theta}^{2}}{2}=\frac{1}{6}\left(-u^{3}+3 D u^{2}+6 c_{1} u+6 c_{2}\right)=\frac{P(u)}{6},
$$

where $P(u)=-u^{3}+3 D u^{2}+6 c_{1} u+6 c_{2}$. Then, the averaged Lagrangian (28) becomes

$$
\overline{\mathcal{L}} \approx \frac{2 k}{\sqrt{3}} \sqrt{D} \int_{u_{2}}^{u_{3}} \sqrt{P(u)} d u-c_{1} \beta-c_{2}+\frac{\beta(\gamma-D \beta)}{2}, \quad D=\omega / k .
$$

The variation with respect to $c_{2}$ gives us the dispersion relation (which is equivalent to the expression (14) for the wave length ):

$$
\frac{1}{k}=2 \sqrt{3 D} \int_{u_{2}}^{u_{3}} \frac{d u}{\sqrt{P(u)}}
$$

The variation with respect to $c_{1}$ gives us the identity $\beta=\bar{u}$. Finally, the last two Euler-Lagrange equations

$$
\begin{aligned}
& \left(\overline{\mathcal{L}}_{\gamma}\right)_{t}-\left(\overline{\mathcal{L}}_{\beta}\right)_{x}=0, \\
& \left(\overline{\mathcal{L}}_{\omega}\right)_{t}-\left(\overline{\mathcal{L}}_{k}\right)_{x}=0,
\end{aligned}
$$

should be written. The equation (29) is exactly equation (23) :

$$
\bar{u}_{t}+\left(\frac{\overline{u^{2}}}{2}\right)_{x}=0
$$

Its combination with the equation $\beta_{t}+\gamma_{x}=0$ gives us $c_{1}=\frac{\gamma-D \beta}{2}$. One also has :

$$
\overline{\mathcal{L}}_{\omega}=\frac{\overline{u^{2}}-(\bar{u})^{2}}{2 k}+\frac{\bar{P}}{6 D k}, \quad \overline{\mathcal{L}}_{k}=-D\left(\frac{\overline{u^{2}}-(\bar{u})^{2}}{2 k}-\frac{\bar{P}}{6 D k}\right) .
$$

Hence the wave action equation (30) is :

$$
\left(\frac{\overline{u^{2}}-(\bar{u})^{2}}{2 k}+\frac{\bar{P}}{6 D k}\right)_{t}+\left(D\left(\frac{\overline{u^{2}}-(\bar{u})^{2}}{2 k}-\frac{\bar{P}}{6 D k}\right)\right)_{x}=0 .
$$

The equation (31) can also be obtained as a consequence of the equations (23), (24), (25) [33]. 


\section{Solitary limit}

The solitary limit is a singular limit of the modulation equations when the wave length $L \rightarrow \infty$ (or $k \rightarrow 0$, or $m \rightarrow 1$ ). In this limit, one has

$$
\overline{u^{2}} \rightarrow \bar{u}^{2}, \quad \overline{P(u)} \rightarrow P(\bar{u})=0 .
$$

Thus, the equations (23)-(24) have the same limit:

$$
\bar{u}_{t}+\bar{u} \bar{u}_{x}=0 .
$$

We need thus to find the limit form of (31). The simplest way to compute the limit, is to use the Wolfram Mathematica because the hand calculations are feasible but a bit painful. One obtains the following a little bit ugly equation :

$$
\left(\frac{a^{3 / 2}(2 a+5 \bar{u})}{\sqrt{a+3 \bar{u}}}\right)_{t}+\left(\frac{a^{3 / 2}(4 a+15 \bar{u}) \sqrt{a+3 \bar{u}}}{9}\right)_{x}=0 .
$$

The final conservative system for the solitary limit of the BBM equation is thus

$$
\begin{aligned}
& \bar{u}_{t}+\left(\frac{(\bar{u})^{2}}{2}\right)_{x}=0, \\
& F(a, \bar{u})_{t}+G(a, \bar{u})_{x}=0,
\end{aligned}
$$

where

$$
F(a, \bar{u})=\frac{a^{3 / 2}(2 a+5 \bar{u})}{\sqrt{a+3 \bar{u}}}, \quad G(a, \bar{u})=\frac{a^{3 / 2}(4 a+15 \bar{u}) \sqrt{a+3 \bar{u}}}{9}
$$

The quasilinear form of (32) is :

$$
\bar{u}_{t}+\bar{u} \bar{u}_{x}=0, \quad a_{t}+D a_{x}+\frac{a}{3} \frac{14 a^{2}+75 a \bar{u}+90 \bar{u}^{2}}{8 a^{2}+40 a \bar{u}+45 \bar{u}^{2}} \bar{u}_{x}=0, \quad D=\bar{u}+\frac{a}{3} .
$$

The characteristics of this hyperbolic system are $\bar{u}$ and $D$. We will construct now closed form non-stationary solutions of (33).

\section{Interaction of the solitary waves with a step}

Consider the Cauchy problem for (32):

$$
(\bar{u}, a)(0, x)= \begin{cases}\left(u^{-}, a^{-}\right), & x<0 \\ \left(u^{+}, a^{+}\right), & x>0 .\end{cases}
$$

We are looking for self-similar continuous solutions of (32) (or (33)) for the corresponding Riemann problem in the case $0<u^{-}<u^{+}$(the case of "positive" rarefaction waves). In particular, the simple wave solution of this system can be interpreted as the interaction of an incident solitary wave of amplitude $a^{-}$with 


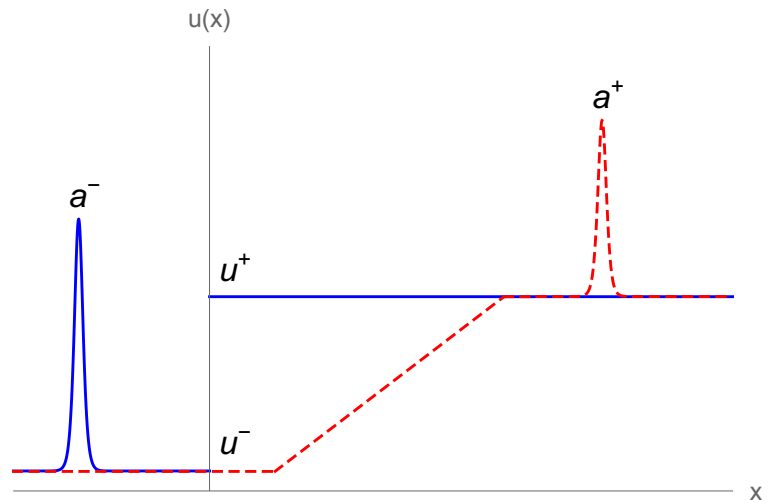

Figure 1: A sketch of the interaction of solitary wave of amplitude $a^{-}$with a step. The solitary wave of amplitude $a^{+}$can exist only for $a^{-}$greater than some critical value.

a step function. As a result of such an interaction, an outgoing solitary wave of amplitude $a^{+}$is formed (see Figure 1). Such a problem was, in particular, studied numerically in [8]. We obtain here its analytical solution.

The Hopf equation implies : $\bar{u}=s=x / t, u^{-}<s<u^{+}$. For the function $a(s)=a(\bar{u})$ one obtains the following ODE :

$$
\frac{d a}{d \bar{u}}=-\frac{G_{\bar{u}}-\bar{u} F_{\bar{u}}}{G_{a}-\bar{u} F_{a}}=-\frac{14 a^{2}+75 a \bar{u}+90 \bar{u}^{2}}{8 a^{2}+40 a \bar{u}+45 \bar{u}^{2}} .
$$

It admits the group transformation $a \rightarrow b a, \bar{u} \rightarrow b \bar{u}, b=$ const. For the corresponding invariant $z=a / \bar{u}$ one obtains the equation

$$
\bar{u} \frac{d z}{d \bar{u}}=-f(z), \quad f(z)=\frac{14 z^{2}+75 z+90}{8 z^{2}+40 z+45}+z .
$$

It allows us to obtain the relation between the incoming $a^{-}$and outgoing $a^{+}$ solitary wave amplitudes :

$$
\int_{z^{+}}^{z^{-}} \frac{d z}{f(z)}=\ln \frac{u^{+}}{u^{-}}, \quad z^{ \pm}=\frac{a^{ \pm}}{u^{ \pm}}
$$

The relation (35) can be written as

$$
p\left(z^{-}\right)-p\left(z^{+}\right)=\ln \left(\frac{u^{+}}{u^{-}}\right), \quad z^{ \pm}=\frac{a^{ \pm}}{u^{ \pm}},
$$

with

$$
p(z)=\frac{1}{24}\left(-2 \sqrt{15} \operatorname{Arctan}\left(\frac{15+8 z}{\sqrt{15}}\right)-6 \ln (3+z)+15 \ln \left(15+15 z+4 z^{2}\right)\right) .
$$


The condition for the solitary wave trapping is $z^{+}=0$. To have a solitary wave which is capable to pass the initial step function, we have to take $z^{-}$larger than the minimal value $z_{\min }^{-}$which is a unique root of the equation

$$
p\left(z_{\min }^{-}\right)-p(0)-\ln \left(\frac{u^{+}}{u^{-}}\right)=0 .
$$

The relation (36) between incoming-outgoing amplitudes $a^{ \pm}$is shown in Figure 2 for particular values of $u^{ \pm}, u^{-}<u^{+}$. In Figure 3 we show the comparison of the theoretical curve and numerical results for the exact BBM equation ("dots") for different values of the incoming amplitude $a^{+}$. The maximum of the initial solitary wave was placed at $x_{0}=-400$, the initial discontinuity was replaced by the hyperbolic tangent :

$$
u(0, x)=u^{+}+\left(u^{+}-u^{-}\right) \tanh \left(\frac{x-x_{0}}{l}\right),
$$

with $l=100$. The numerical results do not depend on the choice of $x_{0}$ and $l$, if $x_{0} \gg l \gg 1$. A very good agreement between the theoretical and numerical results can be observed. In Figure 4 a solitary wave having the incoming amplitude $a^{-} \approx 2.24813$ is taken. For $u^{-}=1 / 3$ and $u^{+}=1$ the amplitude $a^{+}$ of the outgoing wave fits perfectly the theoretical value $a^{+}=1$. In the case of several solitary waves having the same amplitude $a^{-}$one obtains the solitary wave train of the same amplitude $a^{+}$.

One can also remark that the equations (32) can be rewritten in terms of the Riemann invariants :

$$
\bar{u}_{t}+\bar{u} \bar{u}_{x}=0, \quad r_{t}+D r_{x}=0, \quad D=\bar{u}+\frac{a}{3},
$$

with

$$
r=\ln (\bar{u})+p\left(\frac{a}{\bar{u}}\right)
$$

where $p(z)$ is given by (37). Thus, the condition (36) is the conservation of the Riemann invariant $r$.

\section{Generalized Riemann problem for dispersive equations}

We call a generalized Riemann problem (GRP) the Cauchy problem

$$
u(0, x)=\left\{\begin{array}{l}
u_{L}(x), x<0, \\
u_{R}(x), x>0,
\end{array}\right.
$$

where $u_{L, R}(x)$, are different periodic travelling wave solutions of the corresponding dispersive equations (in particular, of the BBM equation). Such a problem was studied in [14] for the SGN equations and Boussinesq equations with linear 


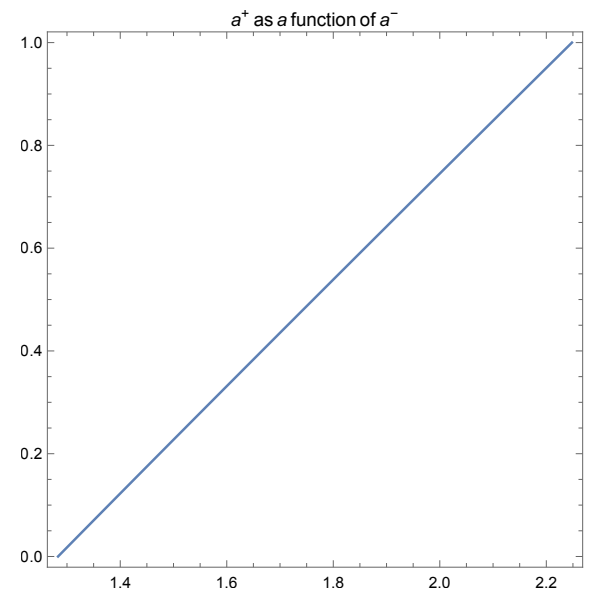

Figure 2: The case $u^{-}=1 / 3$ and $u^{+}=1$ is illustrated. The amplitude $a^{+} \in$ $[0,1]$ of the outgoing solitary wave as a function of the incoming wave amplitude $a^{-}$is shown. In particular, the condition (38) for the wave trapping $\left(a^{+}=0\right)$ gives us $a^{-} \approx 1.283212944$. To have $a^{+}=1$ we need to take $a^{-} \approx 2.24813144$ (for this, one needs to solve (36)).

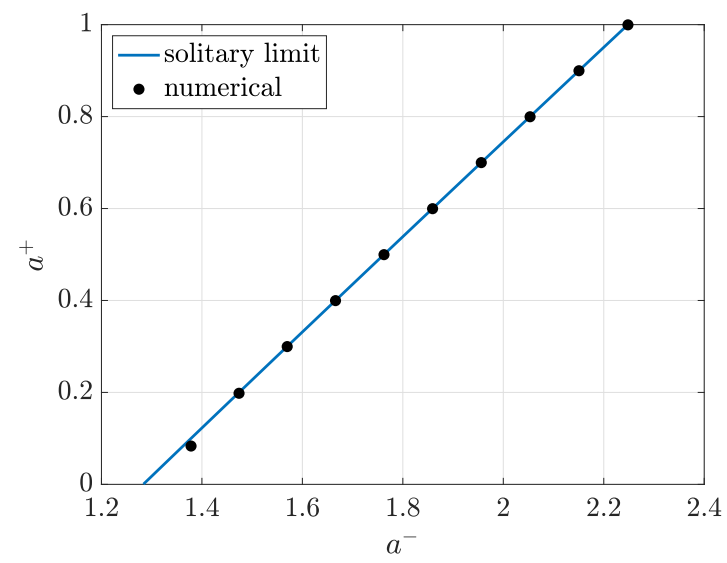

Figure 3: The case $u^{-}=1 / 3$ and $u^{+}=1$ is illustrated. The theoretical relation (36)-(37) (continuous line) is compared with the corresponding numerical computations for the exact BBM equation (shown by "dots"). A very good agreement is observed. 


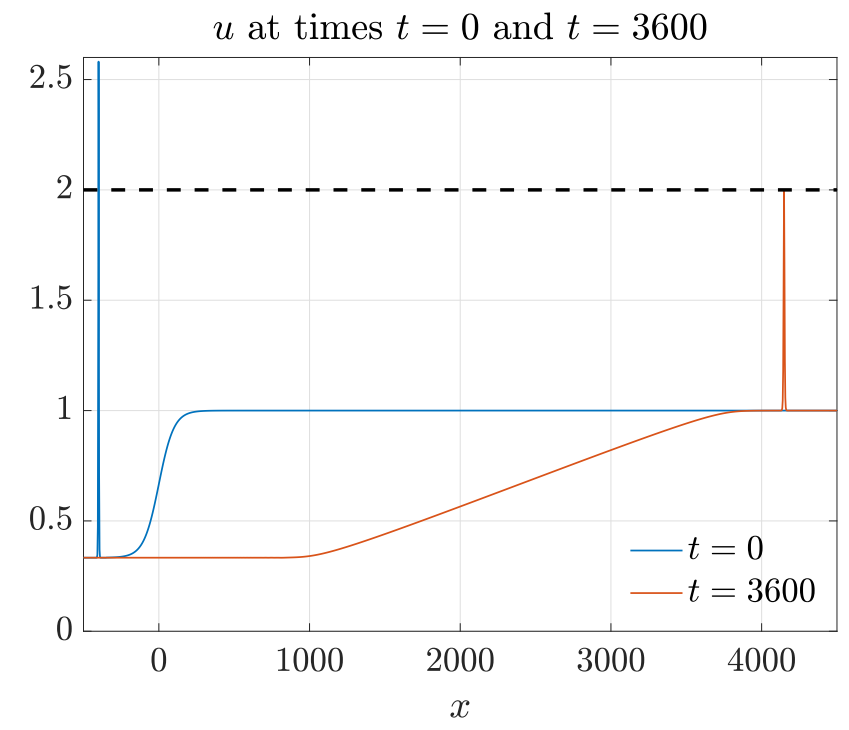

Figure 4: The incoming solitary wave of amplitude $a^{-} \approx 2.24813$ produces the outgoing solitary wave of amplitude $a^{+}=1$ (for $u^{-}=1 / 3$ and $u^{+}=1$ ).

dispersion, and in [38] for the fifth order $\mathrm{KdV}$ equation. In particular, in the first reference new stable shock-like travelling wave solutions were found linking a constant solution with a periodic wave train. The shock-like transition zone between the constant state and the wave train was well described by the half of solitary wave having the wave crest at the maximum of the nearest periodic wave. Let us consider the BBM equation in the form

$$
u_{t}+u u_{x}-\varepsilon^{2} u_{t x x}=0,
$$

where $\varepsilon$ is a small dispersion parameter characterizing the transition zone scale. When $\varepsilon \rightarrow 0$ the half of solitary wave becomes a singular shock on which the RH conditions are satisfied [14]. Such a configuration was stable under certain conditions. The aim of this section is to describe in details the analogous solutions for the BBM equations and propose an explicit criterion for the existence of such stable solutions.

For numerical purposes, we restrict our attention to a modified version of (41) in the form

$$
u(0, x)=\left\{\begin{array}{l}
u(x), x_{0}<x<x_{1}, \\
\bar{u}, \text { if } x \text { is outside of }\left(x_{0}, x_{1}\right) .
\end{array}\right.
$$

Here $\left(x_{0}, x_{1}\right)$ is the interval which contains a quite large number of entire periods (see Figure 5). Indeed, since the BBM equation has a "hyperbolic" structure (the waves propagate with a finite speed), it is much easier to apply the numerical method developed for the BBM equation when the solution tends to a 


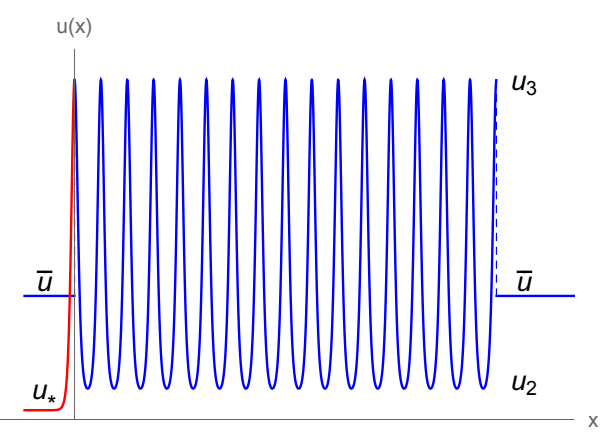

Figure 5: Skecth of the initial configuration (42) consisting of a periodic wave train having the property $D \geq \bar{u}$ and bounded on the left and on the right by the constant state $\bar{u}$. If, initially, instead of $\bar{u}$, one puts on the left the state $u_{\star}^{-}$connected with the wave train by the half-solitary wave (red curve), the left boundary of the wave train remains invariable in time.

constant value at infinity (see a short description of the method in Appendix B). The solution of such a Cauchy problem will show on the left of the periodic wave train of a stable configuration consisting of a "cold" (constant) state which is linked with the "hot" state (the periodic wave train).

\subsection{Generalized RH conditions for the BBM equation and shock conditions for the Whitham system}

Travelling wave solution $u(x)$ for the BBM equation is a smooth extremal curve of the functional

$$
a[u]=\int \mathcal{L}\left(u, u^{\prime}\right) d x, \quad \mathcal{L}\left(u, u^{\prime}\right)=\frac{D u^{\prime 2}}{2}+\frac{P(u)}{6},
$$

where the third order polynomial $P(u)$ is given by $(10)$, and the integral is taken over the basic period of $u(x)$. The variation of $a$ can be written as :

$$
\delta a=\int\left(\frac{\delta \mathcal{L}}{\delta u} \delta u+\frac{d}{d x}\left(\frac{\partial L}{\partial u^{\prime}} \delta u\right)\right) d x, \quad \frac{\delta \mathcal{L}}{\delta u}=\frac{\partial \mathcal{L}}{\partial u}-\frac{d}{d x}\left(\frac{\partial \mathcal{L}}{\partial u^{\prime}}\right) .
$$

In the case of non-smooth ("broken") extremal curves, the Weierstarss-Erdmann condition should be satisfied at the "broken" point $[16]: \frac{\partial \mathcal{L}}{\partial u^{\prime}}=D u^{\prime}$ is continuous. In particular, if a piecewise $C^{2}$-solution $u(x)$ is constant on some interval of $x$, but is not constant on a neighboring interval, this last should have a zero slope at the "broken" point. Thus, the classical Rankine-Hugoniot condition based on the conservation laws should be supplemented by this additional condition. Such weak solutions describing shock-like transition fronts have been constructed for the SGN equations [14]. 
For the BBM equation, we also look for a possibility to link a generic constant state ( "cold" state) $u_{\star}$ with a generic periodic wave train ("hot" state) by the Rankine-Hugoniot conditions through the shock front having the same velocity as the phase velocity $D$ of the wave train (see Figure 5).

For the conservation law (2), the generalized Rankine-Hugoniot (GRH) condition on the travelling wave solutions connecting the constant state $u_{\star}$ and travelling wave train is :

$$
-D\left(u_{3}-\left.u^{\prime \prime}\right|_{u=u_{3}}-u_{\star}\right)+\left(\frac{u_{3}^{2}}{2}-\frac{u_{\star}^{2}}{2}\right)=0 .
$$

The numerical study shows that in the case of positive $u$, the state $u_{\star}$ is linked with the maximum of the travelling wave, i.e., $u_{3}$, and not with the minimum, i.e., $u_{2}$. The equation for $u_{\star}$ is a second order algebraic equation which has two real roots, $u_{\star}^{ \pm}, 0 \leq u_{1}<u_{\star}^{-}<u_{2}<\bar{u}<u_{\star}^{+}<u_{3}$. Replacing the second derivative at $u=u_{3}$, the GRH condition can be written also as

$$
-D\left(u_{3}+\frac{\left(u_{3}-u_{1}\right)\left(u_{3}-u_{2}\right)}{2\left(u_{1}+u_{2}+u_{3}\right)}-u_{\star}\right)+\left(\frac{u_{3}^{2}}{2}-\frac{u_{\star}^{2}}{2}\right)=0 .
$$

The reason to choose the conservation law (2), and not (3) or (4), is justified as follows.

Proposition The solutions $u_{\star}$ obtained from both, the $R H$ condition coming from the Whitham system

$$
-D\left(\bar{u}-u_{\star}\right)+\left(\frac{\overline{u^{2}}}{2}-\frac{u_{\star}^{2}}{2}\right)=0,
$$

and the GRH condition given by (45) (or (46)) coincide.

Proof Subtracting (47) from (46), one obtains :

$$
-D\left(u_{3}-\bar{u}\right)+\left(\frac{u_{3}^{2}}{2}-\frac{\overline{u^{2}}}{2}\right)=\frac{\left(u_{3}-u_{1}\right)\left(u_{3}-u_{2}\right)}{6} .
$$

It is sufficient to prove that this is an identity. To show this, one can use the inverse formulas (19) for $u_{1}, u_{2}, u_{3}$ and (26b) for $\overline{u^{2}}$ to express them in terms of $\bar{u}, a$ and $m$. Then the proof is direct.

As we have already mentioned, there are two roots $u_{\star}^{ \pm}$describing "cold" states and given by (46) (or (47)). The state $u_{\star}^{+}$does not correspond to a stable solution, only the state $u_{\star}^{-}$appears in the numerical solution of the corresponding Cauchy problem. This is why we will further use the notation $u_{\star}$ as a shorter notation for $u_{\star}^{-}$. When the Cauchy problem (42) is solved, the "cold" state $u_{\star}$ is rapidly formed on the left of the periodic wave train. If the wave train length would be infinite, it would be a weak solution to the BBM equation defined on the whole real axis. The only question is the stability of such a configuration. 


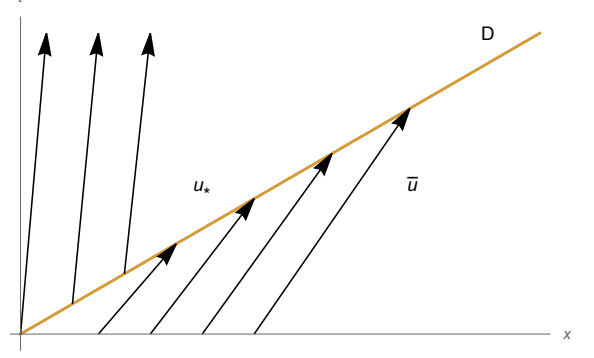

Figure 6: The relation between the characteristics of the homogeneous states $u_{\star}$ and $\bar{u}$ and the shock front having the velocity $D$.

\subsection{Stable shock-like transition fronts}

The numerical experiments show that the condition for the formation of such a stable configuration is $D \geq \bar{u}$, i.e., the phase velocity is not less than the characteristic velocity $\bar{u}$ of the wave train considered as a homogeneous state $\bar{u}$ (see Figure 7). In this case the constant state $u_{\star}$ rapidly forms, and the wave train is not at all perturbed on the left : such a configuration is stable. As in [14], one can numerically show show that if, initially, we take on the left of the wave train the state $u_{\star}^{-}$instead of $\bar{u}$ and smooth the transition zone by the half of solitary wave (see Figure 5) this structure remains invariable in time. If, at the beginning, such smoothing is not performed, after a non-stationary transient process, such a half-soliton structure is quickly established.

The limit case $D=\bar{u}$ can be seen as the analogue of the Chapman-Jouget condition in the detonation theory. This equality can be expressed as $m=m_{c} \approx$ 0.961149 (see (22)). The inequality $D \geq \bar{u}$ is equivalent to $m \geq m_{c}$. Physically, this means that the periodic waves should be "almost" solitary waves. Indeed, for the solitary waves their phase velocity is given by the formula $D=\bar{u}+\frac{a}{3}$, i.e., $D>\bar{u}$ is equivalent to $a>0$. The mathematical reason for the stability is probably the following. Since the shock velocity coincides with the phase velocity, i.e., it is given a priori, it is sufficient to have just one characteristic entering the shock, so no need to satisfy the Lax condition. If $m$ is outside the interval $\left[m_{c}, 1\right)$ (i.e. $D<\bar{u}$ ), such a stationary shock-like configuration on the left does not exist (see Figure 8). The "cold" state appears separating the classical dispersive shock (on the left) and wave train, but the wave train is destroyed by the rarefaction waves coming from the left and from the right of the wave train.

Finally, for such a stable configuration, we are also able to determine the amplitude of the leading right solitary wave which is emitted on the right by the periodic wave train of finite length. The answer is surprisingly simple. Even if we cannot rigorously explain the mathematical reason of this, we can give an analytical expression for the amplitude of the leading solitary wave. Recall again that if the periodic wave train has the property $1>m \geq m_{c}$ (or, what is 

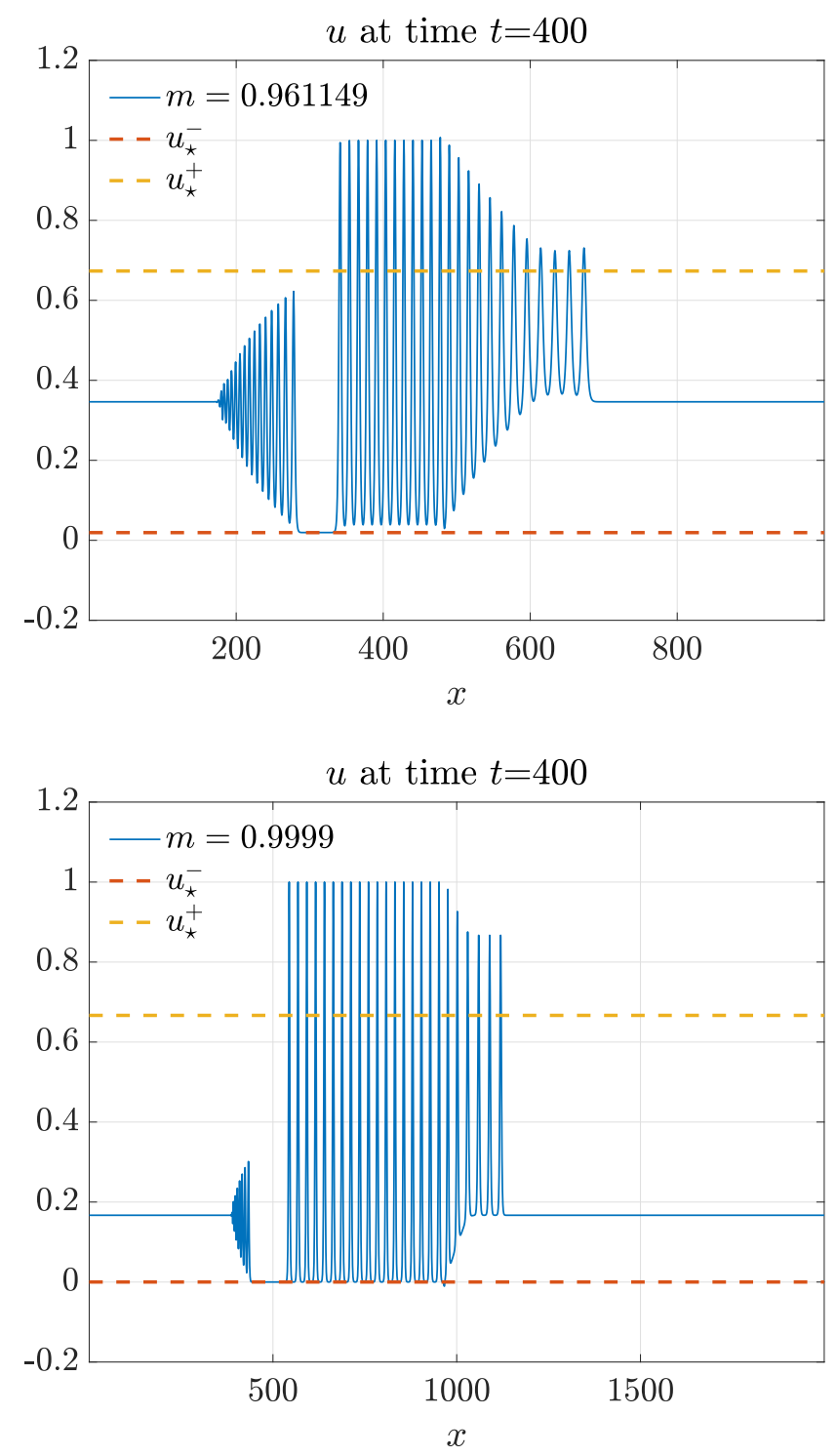

Figure 7: Initially, we consider a periodic wave train with $m \in\left[m_{c}, 1\right)$, with the constant states $\bar{u}$ on the left and on the right. We have chosen $u_{1}=0, u_{3}=1$ and $u_{2}=u_{3}(1-m)$, with parameter $m \in\left[m_{c}, 1\right)$. Then, on the left, a cold state $u_{\star}<u_{2}$ is formed connecting by the generalized RH condition (45) with the periodic wave train. Here the lower (upper) dashed line corresponds to the value $u_{\star}^{-}\left(u_{\star}^{+}\right)$. Such a configuration linking the "star" state with the wave train is stable. 


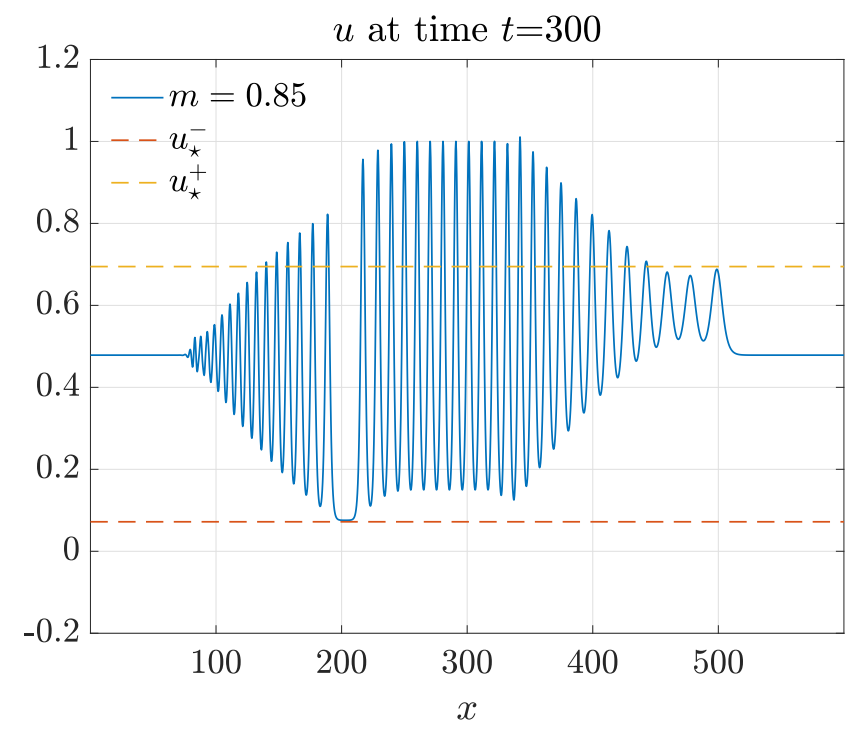

Figure 8: A periodic wave train with $u_{1}=0, u_{3}=1, u_{2}=u_{3}(1-m)$ and $m=0.85<m_{c}$ is taken, bounded by the constant states $\bar{u}$ on the left and on the right. The "cold" state is formed but the periodic wave train represents only a transient structure : it is destroyed by the rarefaction waves.

equivalent, its travelling velocity is not less than $\bar{u}$ ), there exist a "cold" state $u_{\star}, u_{1}<u_{\star}<u_{2}<\bar{u}<u_{3}$ such that the wave train is connected with the "cold" state on the left by the half of a solitary wave having the amplitude $a_{s}^{-}=u_{3}-u_{\star}$. Now, we claim that to define the amplitude of the solitary wave $a_{s}^{+}$on the right, it is sufficient to solve the equation (36) :

$$
p\left(z^{-}\right)-p\left(z^{+}\right)-\ln \left(\frac{u^{+}}{u^{-}}\right)=0,
$$

with

$$
z^{-}=\frac{a_{s}^{-}}{u^{-}}, \quad u^{-}=u_{\star}, \quad a_{s}^{-}=u_{3}-u_{\star}, \quad z^{+}=\frac{a_{s}^{+}}{u^{+}}, \quad u^{+}=\bar{u} .
$$

expressing the condition $r=$ const. In other words, if one takes the incident solitary wave of amplitude $u_{3}-u_{\star}$ (and not as $u_{3}-u_{2}$ ), one obtains the leading solitary wave emitted by the wave train of amplitude $a^{+}$defined by (48). This rather unexpected result is in very good agreement with the numerical results obtained by solving the corresponding Cauchy problem for the BBM equation (see Figure 9). In particular, for $u_{1}=0, u_{3}=1$ and $u_{2}=(1-m)$ one has the following approximate values : for $m=0.9999$ one obtains $\bar{u} \approx 0.166946$, $u_{\star} \approx 0.00005, a^{-}=1-u_{\star}$, and finally $a^{+} \approx 0.699956, D \approx 0.400265$; for $m=m_{c} \approx 0.961149$, one has $\bar{u} \approx 0.346284, u_{\star} \approx 0.019233, a^{-}=1-u_{\star}$, and 

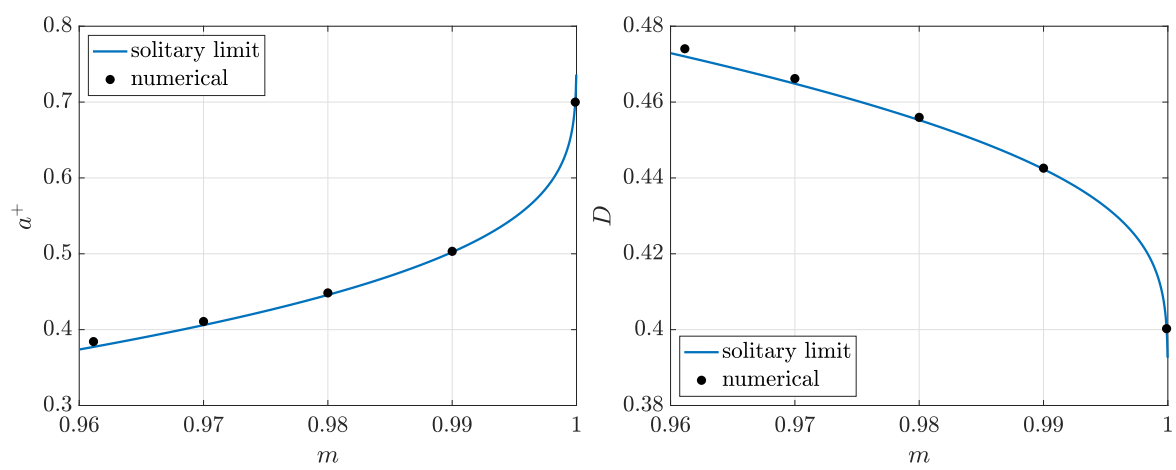

Figure 9: The amplitude and phase velocity of the right leading solitary wave emitted by $L$-periodic wave train $(u(0, x)=u(0, x+L))$ of finite length bounded by the constant states $\bar{u}$ on the left and on the right. The dots are numerical solutions of the Cauchy problem (42) for the BBM equation corresponding to $u_{1}=0, u_{3}=1, u_{2}=(1-m)$, for $m=m_{c} \approx 0.961149,0.97,0.98,0.99$ and 0.9999 .

finally $a^{+} \approx 0.37728, D \approx 0.4720$. We find good agreement between analytical results and numerical solutions (see Figure 9 ).

\section{Conclusion}

The existence of stable shock-like transition fronts linking a constant state with a periodic wave train was discovered in [14] for the SGN equations. Here we have established the analogous result for the BBM equation which shares with the SGN equations the same property of finite phase and group velocity for the corresponding linearized equations. The front represents the half of solitary wave linking the constant state with the periodic wave train. We formulate the condition for existence of such a shock-like structure : the phase velocity of the periodic wave train should be not less than its average value.

The solitary limit of the Whitham modulation equations was derived. The equations of the solitary limit are hyperbolic and admit the Riemann invariants in explicit form. This allowed us, in particular, to test the numerical method for the BBM equation on asymptotically exact solutions. For a special Cauchy problem (42), the amplitude of the right leading solitary wave has been explicitly determined by (48) which is the Riemann invariant of the hyperbolic system (40) describing the solitary limit.

Acknowledgments The authors thank G. El and M. Pavlov for helpful suggestions and discussion. 


\section{A Useful expressions}

The expressions of $A_{i}[m]$ and $P_{2}[m]$ can also be given in terms of the complete elliptic integrals $K[m]$ and $E[m]$ :

$$
\begin{aligned}
& A_{1}(m)=\frac{E(m)-(1-m) K(m)}{m K(m)}, \\
& A_{2}(m)=\frac{(-2+4 m) E(m)+(2-3 m)(1-m) K(m)}{3 m^{2} K(m)} \\
& A_{3}(m)=\frac{(8+23 m(m-1)) E(m)+(-8+m(19-15 m))(1-m) K(m)}{15 m^{3} K(m)}, \\
& P_{2}(m)=\frac{2(1+m(m-1)) E(m)+(-2+m)(1-m) K(m)}{15 m^{2} K(m)} .
\end{aligned}
$$

The formulas are useful to compute approximate theoretical values of the phase velocity $D, \bar{u}$, and so on, by using Wolfram Mathematica, for example.

\section{B Numerical method}

To find approximate solutions to the BBM equation, we use the hyperbolicelliptic splitting approach developed previously in $[28,14]$. This algorithm consists of two steps. In the first step, the hyperbolic step, we employ the state-ofthe-art method for hyperbolic conservation laws for the numerical resolution of the equation

$$
\mathcal{K}_{t}+\left(\frac{u^{2}}{2}\right)_{x}=0, \quad \text { with } \quad \mathcal{K}=u-u_{x x},
$$

over a time step $\Delta t$. In the second step, the elliptic step, using the approximate solution $\mathcal{K}$ computed during the hyperbolic step, we invert numerically the elliptic operator:

$$
u-u_{x x}=\mathcal{K}
$$

with prescribed boundary conditions based on a fourth-order compact scheme [26].

More precisely, in the hyperbolic step, we use the semi-discrete finite volume method written in a wave-propagation form as before [14], but employ a different solution reconstruction technique, the BVD (boundary variation diminishing) principle, which is more robust than the classical one for the interpolated states ( $\mathcal{K}$ for the BBM equation) at cell boundaries (cf. [9] and the references cited therein). These reconstructed variables form the basis for the initial data of the Riemann problems, where the solutions of the Riemann problems (obtained from the local Lax-Friedrichs approximate solver [27] for the BBM equation) are then used to construct the fluctuations in the spatial discretization that gives the right-hand side of the system of ODEs (cf. [24, 25]). To integrate the ODE system in time, the strong stability-preserving (SSP) multistage Runge-Kutta scheme $[22,37]$ is used. In particular, for the numerical results presented in this paper, the third-order SSP scheme was employed together with the pair of 
third- and fifth-order WENO (weighted essentially non-oscillatory) scheme in the BVD reconstruction process.

\section{References}

[1] Abramowitz M and Stegun I A 1964 Handbook of Mathematical Functions with Formulas, Graphs, and Mathematical Tables, National Bureau of Standards Applied Mathematics Series, No. 55, U. S. Government Printing Office, Washington, D. C.

[2] Bona J L and Tzvetkov N 2009 Sharp well-posedness results for the BBM equation, Discrete and Continuous Dynamical Systems 2341241 - 1252.

[3] Bakholdin I B 2004 Non-dissipative discontinuities in continuum mechanics, Fizmatlit (in Russian).

[4] Bakholdin I B 2021 Analysis of the equations of two-fluid plasma in the approximation of electro-magneto hydrodynamics and the structure of discontinuities of their solutions, J. Comp. Math. and Math. Physics 61 458-474 (in Russian).

[5] Benjamin T B, Bona J L and Mahony J J 1972 Model equations for long waves in nonlinear dspersive systems. Phil. Trans. Royal Soc. A 272 47-78.

[6] Benzoni-Gavage S, Mietka C and Rodrigues L M 2021 Modulated equations of Hamiltonian PDEs and dispersive shocks, Nonlinearity 34578.

[7] Bhatnagar P L 1979 Nonlinear waves in one-dimensional dispersive systems, Clarendon Press, Oxford.

[8] Congy T, El G A, Hoeffer M A and Shearer M 2021 Dispersive Riemann problem for the Benjamin-Bona-Mahony equation https://arxiv.org/abs/2012.14579

[9] Deng X, Inaba S, Xie B, Shyue K M and Xiao F 2018 High fidelity discontinuity-resolving reconstruction for compressible multiphase flows with moving interfaces, J. Comput. Phys. 371 945-966.

[10] El G A, Geogjaev V V, Gurevich A V and Krylov A V 1995 Decay of an initial discontinuity in the defocusing NLS hydrodynamics, Physica D: Nonlinear Phenomena 87 186-192.

[11] El G A, Grimshaw R H J and Smyth N F 2006 Unsteady undular bores in fully nonlinear shallow-water theory, Phys. Fluids 18027104.

[12] El G A and Hoefer M 2016 Dispersive shock waves and modulation theory, Physica D 333 11-65.

[13] El G A, Hoefer M A and Shearer M 2016 Expansion shock waves in regularized shallow water theory, Proc. Royal Soc. A $4 \mathbf{7 2}$.

[14] Gavrilyuk S, Nkonga B, Shyue K M and Truskinovsky L 2020 Stationary shock-like transition fronts in dispersive systems, Nonlinearity 33 54775509 .

[15] Gavrilyuk S and Gouin H 2020 Rankine-Hugoniot conditions for fluids whose energy depends on space and time derivatives of density, Wave motion 98102620. 
[16] Gelfand I M and Fomin S V 2000 Calculus of Variations, Dover.

[17] Gurevich A V, Krylov A V and El G A 1990 Nonlinear modulated waves in dispersive hydrodynamics, J. Exp. Theor. Phys. 98 1605-1626.

[18] Green A E, Laws N and Naghdi P M 1974 On the theory of water waves, Proc. R. Soc. Lond. A 338 43-55.

[19] Green A E and Naghdi P M 1976 A derivation of equations for wave propagation in water of variable depth, J. Fluid Mech. 78 237-246.

[20] Gurevich A, Pitaevskii L 1974 Nonstationary structure of a collisionless shock wave, JETP 38291 - 297.

[21] Gurevich A V and Krylov A V 1987 Dissipationless shock waves in media with positive dispersion, Zh. Eksp. Teor. Fiz. 92 1684-1699.

[22] Gottlieb S, Shu C W and Tadmor E 2001 Strong stability preserving highorder timediscretization methods, SIAM Review 43 89-112.

[23] Kamchatnov A M 2000 Nonlinear Periodic Waves and Their Modulations: An Introductory Course, World Scinetific Publishing.

[24] Ketcheson D I and LeVeque R J 2008 WENOCLAW: A higher order wave propagation method, in: Hyperbolic Problems: Theory, Numerics, Applications, Springer-Verlag, 609-616.

[25] Ketcheson D I, Parsani M and LeVeque R J 2013 High-order wave propagation algorithm for hyperbolic systems, SIAM J. Sci. Comput. 35(1) A351-A377.

[26] Lele S K 1992 Compact finite difference schemes with spectral-like resolution, J. Comput. Phys. 103 16-43.

[27] LeVeque R J 2007 Finite Difference Methods for Ordinary and Partial Differential Equations: Steady-State and Time-Dependent Problems, SIAM, Philadelphia.

[28] Le Métayer O, Gavrilyuk S and Hank S 2010 A numerical scheme for the Green-Naghdi model, J. Comp. Phys. 229 2034-2045.

[29] Mikyoung Hur V and Pandey A K 2016 Modulational instability in nonlinear nonlocal equations of regularized long wave type, Physica D : Nonlinear Phenomena, 325 98-112.

[30] Olver P J 1979 Euler operators and conservation laws of the BBM equation, Math. Proc. Camb. Phil. Soc. 85143 - 160.

[31] Olver P J 1980 On the Hamiltonian structure of evolution equations, Math. Proc. Camb. Phil. Soc. $8871-88$.

[32] Pavlov M 1987 Nonlinear Schrödinger equation and the BogolyubovWhitham method of averaging, Teoreticheskaya Matematicheskaya Fizika $71351-356$.

[33] Pavlov M 2021 Personal communication.

[34] Miles J and Salmon R 1985 Weakly dispersive nonlinear gravity waves, J. Fluid Mechanics 157 519-531.

[35] Pitti J P A, Zoppou C and Roberts S G 2017 Behaviour of the Serre equations in the presence of steep gradients revisited, arXiv:1706.08637v1 [math NA] 27 Jun 2017. 
[36] Serre F 1953 Contribution à l'étude des écoulements permanents et variables dans les canaux, La Houille Blanche 8 374-388.

[37] Shu C W 2009 High order weighted essentially nonoscillatory schemes for convection dominated problems, SIAM Review 5 82-126.

[38] Sprenger P and Hoefer M A 2020 Discontinuous shock solutions of the Whitham modulation equations as dispersionless limits of travelling waves, Nonlinearity 33, 3268-3302.

[39] Tso Taicheng 1992 The zero dispersion limits of nonlinear wave equations ( $\mathrm{PhD}$ thesis)

[40] Whitham G B 1965 Non-linear dispersive waves, Proc. Roy. Soc. London 283 A, 238-291.

[41] Whitham G B 1974 Linear and Nonlinear W-aves, John Wiley and Sons. 